\title{
Overexpression of the alfalfa zeaxanthin epoxidase gene delays seed germination in transgenic tobacco
}

\author{
Y.M. CAO ${ }^{1}$, Z.Q. ZHANG ${ }^{1,2}$, T. ZHANG ${ }^{1}$, J. AN ${ }^{1}$, L.Q. CHANG ${ }^{1}$, Y.F. WANG ${ }^{1}$, H.X. WU ${ }^{3}$, T.M. HU ${ }^{1 *}$, \\ and P.Z. YANG ${ }^{1 *}$ \\ College of Grassland Agriculture, Northwest A\&F University, Yangling, 712100, Shaanxi, P.R. China ${ }^{1}$ \\ College of Grassland, Resources and Environment, Ministry of Agriculture, \\ Inner Mongolia Agricultural University, Hohhot 010011, P.R. China ${ }^{2}$ \\ Grassland Research Institute of CAAS, Hohhot, 010010, Inner Mongolia, P.R. China ${ }^{3}$
}

\begin{abstract}
Zeaxanthin epoxidase (ZEP) plays important roles in plant growth and development due to its functions in abscisic acid (ABA) biosynthesis and in the xanthophyll cycle. Many studies have been exploring the roles of ZEP in seed germination and response to various environmental stresses. In this study, we reported that heterologously overexpressing the $Z E P$ gene from Medicago sativa $(M s Z E P)$ in tobacco increased ABA content in tobacco mature seeds and negatively regulated seed germination. Enhanced ABA synthesis in seed embryo and delayed germination might also be related to the increased 9-cis-epoxycarotenoid dioxygenase (NCED6) expression. Moreover, we found that overexpression of MsZEP resulted in an increased expression of the SOMNUS gene but a decreased expression of the DNA ligase 6 gene (Lig6) suggesting that $M s Z E P$ might affect seed proteome and DNA integrity. Furthermore, enhanced chlorophyll content in transgenic tobacco seedlings overexpressing MsZEP might be due to its function in the xanthophyll cycle and ABA biosynthesis.
\end{abstract}

Additional key words: abscisic acid, chlorophyll, Medicago sativa, Lig6, NCED6, Nicotiana tabacum, SOMNUS, xanthophyll cycle.

\section{Introduction}

Zeaxanthin epoxidase (ZEP) converts zeaxanthin to violaxanthin via the intermediate antheraxanthin and plays important roles in plant growth and development due to its functions in abscisic acid (ABA) biosynthesis and xanthophyll cycle (Nambara and Marion-Poll 2005). The vast majority of recent research attention has been dedicated to exploring the roles of ZEP in response to various environmental stresses in different plant species, including Arabidopsis (Barrero et al. 2005), Nicotiana plumbaginifolia (Xiong and Zhu 2003), Oryza sativa (Agrawal et al. 2001), and Medicago sativa (Zhang et al. 2016).

The ZEP transcription is different among species and tissues under various environment stresses. For example, the amount of $Z E P$ transcripts increases in roots of tobacco and tomato during drought stress, whereas showing little changes in leaves (Thompson et al. 2000a, Agrawal et al. 2001). In alfalfa, MsZEP expression decreases in shoots under drought, cold, heat, and ABA treatments, whereas the expression in roots increases first and then decreases under drought, heat, and ABA treatments (Zhang et al. 2016). The functions of ZEP in plant abiotic stress tolerance have been studied by using transgenic technology in many plants. It was reported that the ZEP gene is involved in the regulation of ABA biosynthesis in roots and contributes to plant response to drought (Audran et al. 2001). Overexpression of the ZEP gene increases sensitivity to high irradiance and chilling stress in tomato (Wang et al. 2008) and enhances salt and drought tolerance in Arabidopsis (Park et al. 2008).

Because of its high vulnerability to biotic and abiotic stresses, germination is the most critical phase in the plant life cycle (Rajjou et al. 2012). Abscisic acid is an important controling factor during seed germination. It is known that ABA promotes seed dormancy and represses seed germination (Holdsworth et al. 2008, Je et al. 2014). The ZEP is the first enzyme that was identified as an ABA biosynthetic enzyme (Marin et al. 1996). Moreover, ABA-

Submitted 15 August 2018, last revision 24 February 2019, accepted 28 February 2019.

Abbreviations: ABA - abscisic acid; Lig6 - DNA ligase 6; MsZEP: - Medicago sativa zeaxanthin epoxidase gene; NCED - 9-cisepoxycarotenoid dioxygenase; SOM - SOMNUS; WT - wild-type; ZEP - zeaxanthin epoxidase.

Acknowledgments: This work was supported by the Project of National Natural Science Foundation of China (31272490; 31572456; 31772660), the China Agriculture Research System (CARS-34), and the Project of National Natural Science Foundation of Inner Mogolia (2017BS0305). Yuman Cao and Zhiqiang Zhang contributed equally to this work.

* Corresponding authors; e-mails: yangpeizhi@126.com, hutianming@126.com 


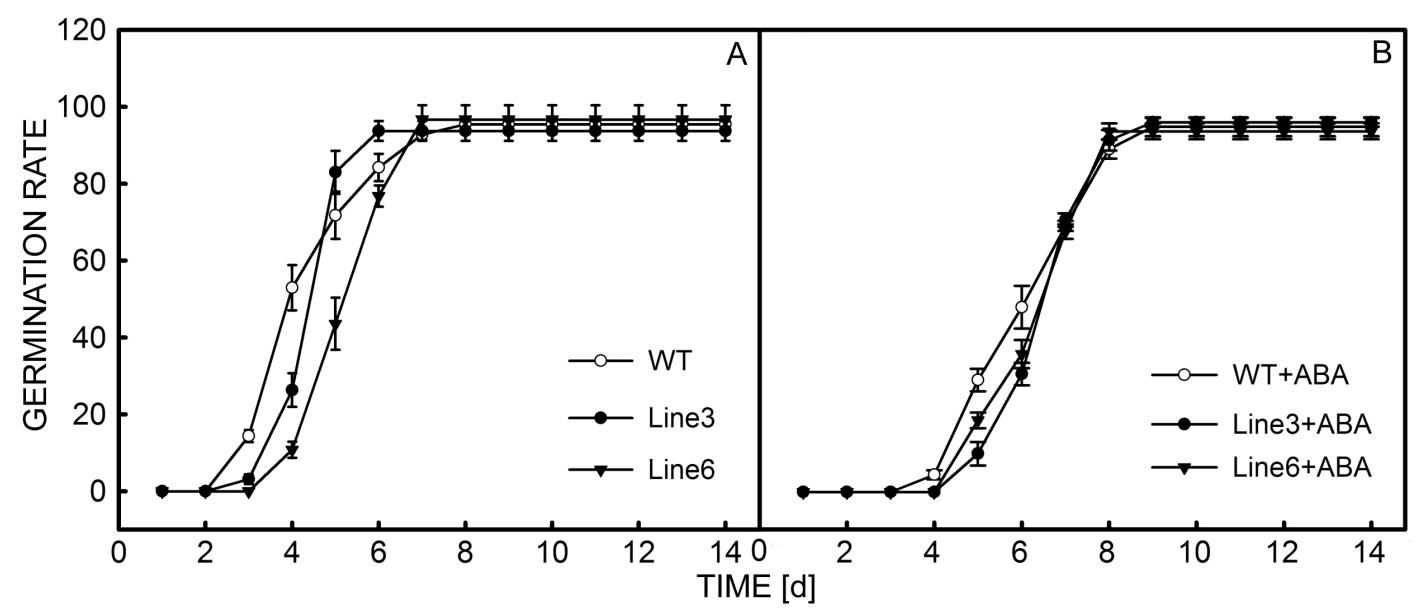

Fig. 1 The effect of MsZEP overexpression on seed germination. Germination rate of wild type (WT) and two MsZEP-overexpressing lines under normal growth conditions $(A)$ and after treatment with $5 \mu \mathrm{MABA}(B)$. Line 3 and Line 6 - two $M s Z E P$-overexpressing plant lines of $\mathrm{T}_{2}$ generation. Means $\pm \mathrm{SEs}, n=3$ sets of seeds, each containing 50 seeds.

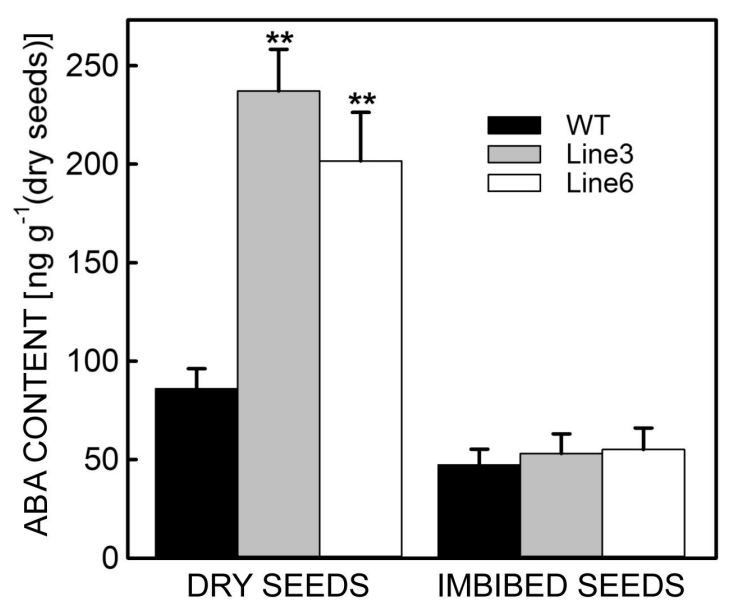

Fig. 2 The effect of $M S Z E P$ overexpression on abscisic acid (ABA) content in tobacco seeds. WT - wild-type tobacco; Line 3 and Line 6 - two MsZEP-overexpressing plant lines of $\mathrm{T}_{2}$ generation. Means \pm SEs, $n=3, *$ and $* *_{-}$differences significant at $P \leq 0.05$ and 0.01 , respectively. deficient mutants, such as Arabidopsis thaliana abal and Nicotiana plumbaginifolia aba2, are defective in zeaxanthin epoxidation (Rock and Zeevaart 1991). Many studies have reported that plants overexpressing a ZEP gene exhibit an increased ABA content in different tissues (Audran et al. 2001, Park et al. 2008, Zhang et al. 2016). The role of ZEP on germination have been previously analyzed in mutants (Marin et al. 1996) and transgenic plants (Frey et al. 1999). It was demonstrated that this gene affects ABA content in seeds and then acts as a negative regulator of germination. An $M s Z E P$ expression is clearly tissuespecific and is highly expressed in green tissues in alfalfa (Zhang et al. 2016). In addition, MsZEP expression is induced by nodulation and abiotic stress treatment. Heterologous expression of MsZEP shows an enhanced ABA content and increased tolerance to drought and salt stresses in tobacco (Zhang et al. 2016). However, little is known about the effect of $M S Z E P$ on seed germination. In this study, we validated the function of $M s Z E P$ during seed germination by heterologous expression of $M s Z E P$ in tobacco.

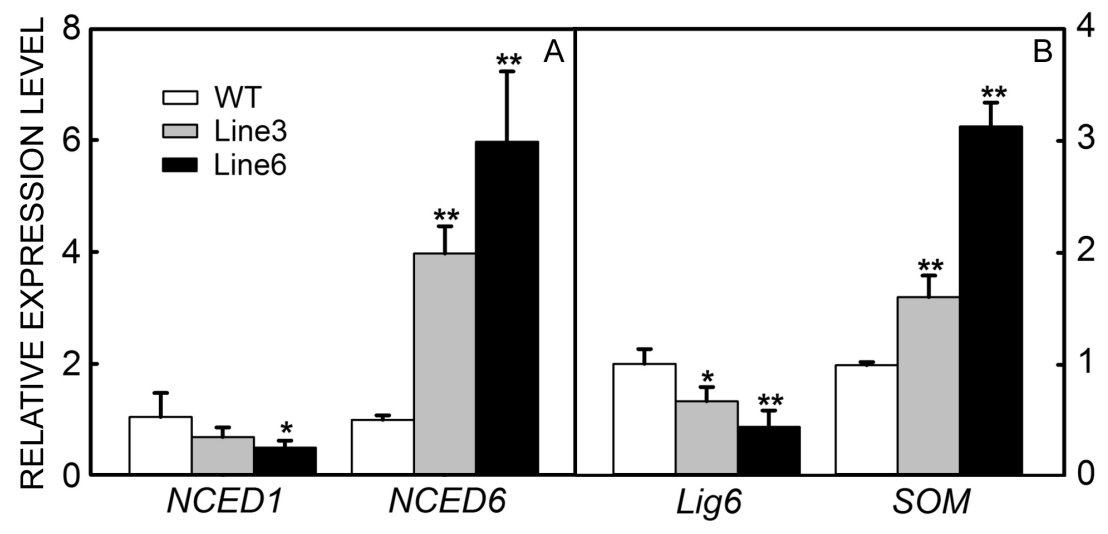

Fig. 3 The effect of $M s Z E P$ overexpression on the expression of genes involved in abscisic acid (ABA) biosynthesis and seed germination. $A$ - The expression of genes involved in ABA biosynthesis in wild type (WT) and MsZEP-overexpressing seeds. $B$ - The expression of genes involved in seed germination in WT and MsZEP-overexpressing seeds. Line 3 and Line 6 - two $\mathrm{T}_{2} M s Z E P$-overexpressing plant lines. Means \pm SEs, $n=3, *$ and $* *$ - differences significant at $P \leq 0.05$ and 0.01 , respectively. 


\section{Materials and methods}

Plants and cultivation: Tobacco (Nicotiana tabacum L.) wild type (WT) and the lines overexpressing $M s Z E P$ controlled by the $35 \mathrm{~S}$ promoter have been previously described (Zhang et al. 2016). To obtain transgenic seeds from these lines, $T_{1}$ tobacco seeds were firstly sown and germinated on a Murashige and Skoog medium containing $30 \mathrm{mg} \mathrm{dm}^{-3}$ hygromycin $\mathrm{B}$ at a temperature of $24^{\circ} \mathrm{C}$, an irradiance of $60 \mu \mathrm{mol} \mathrm{m} \mathrm{m}^{-2} \mathrm{~s}^{-1}$, and a $16-\mathrm{h}$ photoperiod. Subsequently, 10-d-old plantlets were transferred to pots filled with sandy soil in a greenhouse with natural irradiance, an average temperature of $24^{\circ} \mathrm{C}$, and a relative humidity of $65 \pm 5 \%$, and grown till maturity.

Germination assay: Seeds of wild type and $\mathrm{T}_{2}$ transgenic lines were harvested on the same day from tobacco and dried for two weeks. Dried seeds were surfaced sterilized with $70 \%(\mathrm{v} / \mathrm{v})$ ethanol, rinsed with water, and air-dried. Seeds were imbibed on wetted filter paper supplemented with 0 (control) and $5 \mu \mathrm{M} \mathrm{ABA}$ in Petri dishes and then germinated at $24^{\circ} \mathrm{C}$ and a $12-\mathrm{h}$ photoperiod. Experiments were performed in triplicate, with 50 seeds counted for
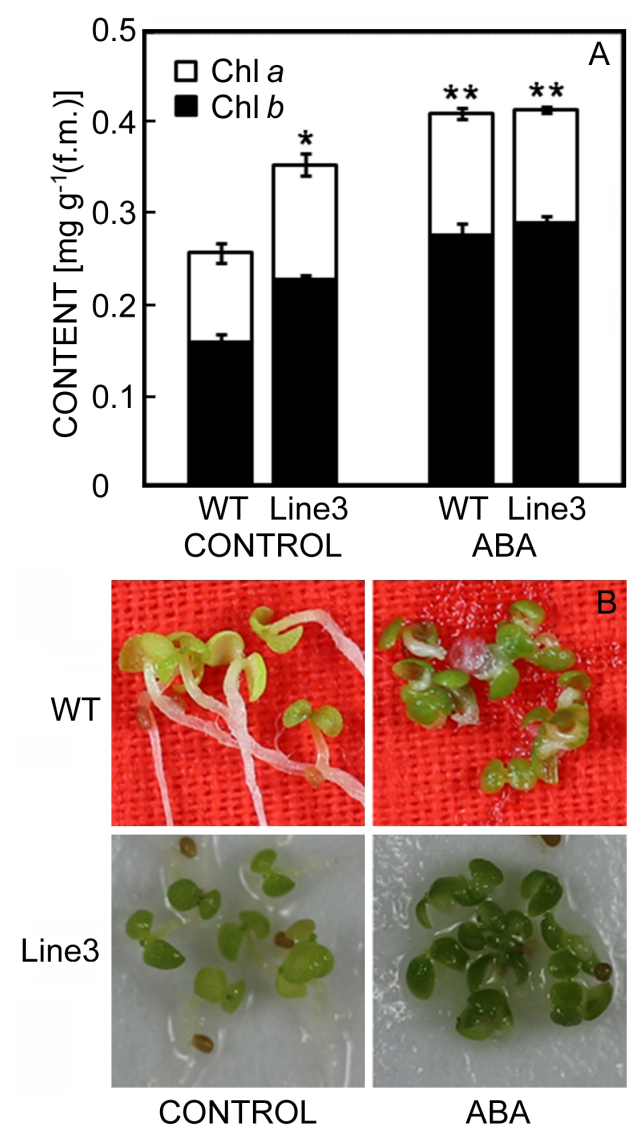

Fig. 4 The effect of MsZEP overexpression on chlorophyll (Chl) content in tobacco seedlings. $A$ - Chlorophyll content in 28-d-old tobacco seedlings germinated under normal conditions and under $5 \mu \mathrm{M}$ ABA treatment. $B$ - Phenotypes of 28-d-old tobacco seedlings. WT - wild-type tobacco; Line $3-\mathrm{T}_{2} M s Z E P$ overexpressing plant line. Means \pm SEs, $n=3$, * and ** differences significant at $P \leq 0.05$ and 0.01 , respectively. each treatment. The number of germinated seeds in each Petri dish was counted every day for 14 days. Seeds with visible radicles were considered as germinated (Rajjou et al. 2012).

Quantification of ABA: Extraction and purification of ABA were carried out according to Park et al. (2008) and Zhang et al. (2016) with some modifications. In brief, about $50 \mathrm{mg}$ of dry tobacco seeds or seeds imbibed in water for $8 \mathrm{~h}$ were frozen in liquid nitrogen and grounded to powder with a tissue lyser (Qiagen, USA), and then the samples were kept in $2 \mathrm{~cm}^{3}$ of $80 \%(\mathrm{v} / \mathrm{v})$ methanol in the dark at $4{ }^{\circ} \mathrm{C}$ overnight. The extract was centrifuged at $4000 \mathrm{~g}$ and $4{ }^{\circ} \mathrm{C}$ for $10 \mathrm{~min}$, and the resulting pellet was re-extracted with another $1 \mathrm{~cm}^{3}$ of $80 \%$ methanol as described above. The supernatants were dried by nitrogen gas and the pellet was re-dissolved in $1 \mathrm{~cm}^{3}$ of methanol, then filtered through a $0.45 \mu \mathrm{m}$ syringe filter. Quantification of hormones by LC-MS/MS was performed as described by Sasaki et al. (2015). Raw values for ABA levels were standardized by plant mass and extraction volume.

Gene expression analysis: Total RNA was extracted from tobacco seeds, and the first strand cDNA was synthesized using a PrimeScript RT reagent kit with a gDNA eraser (Takara, Dalian, China) according to the manufacturer's instructions. Real-time quantitative PCR) was performed as described previously (Zhang et al. 2016). The tobacco actin gene (NtActin) was used as a reference gene. Three independent biological replicates and three technical replicates for each sample were used. The primers are listed in Table 1 Suppl.

Determination of chlorophyll content: Chlorophyll content of the WT and transgenic plants was determined spectrophotometrically using $80 \%(\mathrm{v} / \mathrm{v})$ acetone as a solvent (Harmutk 1987). The absorbance of the extract was measured at 645 and $663 \mathrm{~nm}$ with an Optizen 5100 UV spectrophotometer (Shanghai, China).

Statistical analysis: All data are presented as the means \pm standard errors (SEs) from three biological replicates each containing 50 seeds. Statistical significance was calculated by the Student $t$-test. Analyses were performed with the IBM SPSS Statistics 18.0 software. Figures were created using SigmaPlot 12.5 (Systat software, Germany).

\section{Results and discussion}

Many studies have demonstrated that ABA plays an important role in seed germination (Linkies and LeubnerMetzger 2012, Je et al. 2014). Therefore, we hypothesized that also MsZEP might play an important role in seed germination. We performed a detailed study of the function of $M s Z E P$. Firstly, we examined germination rates of WT and $M s Z E P$-overexpressing seeds under normal conditions. We found that the germination rate of WT was significantly higher than of $M s Z E P$-overexpressing lines on days 3 and $4(P \leq 0.01)$, whereas the germination of WT 
was lower than of line 3 but higher than of line 6 on days 5 and $6(P \leq 0.01)$. These results show that the germination of $M S Z E P$-overexpressing seeds was delayed compared with the WT seeds although the final germination percentage of both seeds was similar (Fig. 1A). For reaching $50 \%$ germination, WT seeds needed about $4 \mathrm{~d}$, whereas transgenic seeds needed 4.5 and $5.5 \mathrm{~d}$ for line 3 and line 6, respectively; the difference between WT and transgenic lines was significant $(P \leq 0.05)$. Under $5 \mu \mathrm{M}$ ABA treatment, the germination of all seeds was delayed, and the $M s Z E P$-overexpressing seeds still geminated slower compared with WT seeds. The germination rate of WT was significantly higher than $M s Z E P$-overexpressing lines between days 4 and $6(P \leq 0.05)$. Wild type seeds reached $50 \%$ germination after around $6 \mathrm{~d}$, whereas overexpressing lines after $6.2 \mathrm{~d}$ (Fig. $1 B$ ).

It is known that ABA promotes seed dormancy and represses seed germination (Holdsworth et al. 2008). Because of the important roles of ZEP in ABA biosynthesis, a higher ABA content was detected in the leaves of $M s Z E P$-overexpressing tobacco than in WT (Zhang et al. 2016). To investigate whether the delayed germination of $M s Z E P$-overexpressing seeds was induced by altered ABA content, ABA content in dry and imbibed seeds was quantified (Fig. 2). Content of ABA in MsZEPoverexpressing dry seeds was significantly higher than in WT $(P \leq 0.01)$. This is consistent with the study showing that the overexpressing NtZEP gene in tobacco under the control of the constitutive $35 \mathrm{~S}$ promoter results in an increase of ABA content in dry seeds and delays seed germination (Frey et al. 1999). However, overexpressing the Arabidopsis AtZEP gene in N. plumbaginifolia plants using seed-specific promoters does not change ABA content in mature seeds (Frey et al. 2006). Since the $35 \mathrm{~S}$ promoter could regulate the $Z E P$ gene in all tissues, the higher ABA content in dry seeds could be due to the translocation from maternal tissues, like leaves, to seeds. When seeds were imbibed in water for $8 \mathrm{~h}$, ABA content decreased rapidly in both WT and transgenic lines, and finally there was no difference among them (Fig. 2). Similarly, Frey et al. (2006) reported that ABA content decreases rapidly and then remains low and stable in imbibed seeds, and there is no difference between WT and AtZEP-overexpressing plants, whereas overexpressing the NtZEP gene could restore seed dormancy but only slightly delays seed germination compared to WT. Therefore, the delayed germination of $M s Z E P$-overexpressing plants might not be caused by the higher ABA content in dry seeds, which might origin from maternal tissues. It was reported that $\mathrm{ABA}$ accumulates firstly in maternal tissues and later in the seed embryo (Groot et al. 1991). Furthermore, reciprocal crosses between WT and ABA-deficient genotypes proved that only ABA produced by the embryo itself, and not maternal ABA, is necessary to impose dormancy (Karrsen et al. 1983). Here, overexpressing the $M s Z E P$ gene under the control of the $35 \mathrm{~S}$ promoter may increase the seed embryo ABA content in normal conditions and even under ABA addition, which delayed the germination of transgenic seeds despite the fact that we cannot distinguish between ABA produced by seed embryos and maternal tissues. It was reported that downstream biosynthetic steps, notably NCED activity, contribute to the ABA synthesis regulation in seed embryos (Lefebvre et al. 2006). Therefore, MsZEP might indirectly regulate seed germination through downstream steps.

Zeaxanthin epoxidase is the first committed enzyme in the ABA biosynthesis pathway, and overexpression of NpZEP increases endogenous ABA content and enhances seed dormancy in tobacco (Cutler and Krochko 1999, Wolters et al. 2010). The NCED catalyze the ratelimiting step in the ABA biosynthesis pathway (Nambara and Marion-Poll 2005), and overexpression of NCED in plants results in higher ABA content and increased seed dormancy (Thompson et al. 2000b, Zhang et al. 2014, Tong et al. 2017). To determine promoting effects of $M s Z E P$ on ABA accumulation, we analyzed the effect of $M s Z E P$ overexpression on the expression of ABA biosynthesis genes in seeds using real-time quantitative PCR. The results show that $M s Z E P$-overexpressing seeds were not significantly different from WT except for ZEP1 in line 3. As concerns NCED, the expressions of NCED6 in $M s Z E P$-overexpressing seeds were significantly higher than those in WT, whereas the expression of NCED1 was lower (Fig. $4 A ; P \leq 0.05$ or $P \leq 0.01$ ). The maintenance of seed dormancy is also affected by the ABA synthesis during seed imbibition (Grappin et al. 2000). The increased expression of NCED6 might contribute to the ABA synthesis in embryos of transgenic seeds during imbibition.

Integrity of DNA and proteome stability play a major role in the germination process (Koornneef et al. 2002). AtLig6 is a major determinant of Arabidopsis seed quality and longevity, and phenotypic analysis reveals a delay in the germination of atlig6 mutants compared with WT lines (Waterworth et al. 2010). SOMNUS (SOM) is a negative regulator of seed germination (Kim et al. 2008). In this study, the effect of MsZEP overexpression on the expression of Lig6 and SOM in seeds was analyzed. The MsZEP-overexpression lines exhibited a decreased Lig6 expression and increased $S O M$ expression compared with WT (Fig. $4 B ; P \leq 0.05$ or $P \leq 0.01$ ). These results suggest that $M s Z E P$ negatively regulating germination might affect seed DNA integrity and proteome stability.

The $M s Z E P$-overexpressing seedlings appeared dark green compared to the light green WT seedlings (Fig. 5B) suggesting that overexpression of the $M s Z E P$ gene may promote plant health. This is consistent with findings that the chlorophyll content of MsZEP-overexpressing seedlings was significantly higher than that of WT under normal conditions (Fig. $5 A ; P \leq 0.05$ ), which may be due to the role of ZEP in xanthophyll cycle. Moreover, the chlorophyll content of both plants increased after ABA treatment $(P \leq 0.01)$. These results suggest that MsZEPoverexpression seedlings contained a higher chlorophyll content, which may be due to the high ABA content.

In summary, we reported that the heterologously overexpressing $M s Z E P$ gene in tobacco increased ABA content in their seeds and negatively regulated seed germination. Enhanced ABA synthesis in seed embryos and delayed germination might also be related to the increased 
NCED6 expression. Also, MsZEP-overexpression in tobacco seed affected cell proteome and DNA integrity as indicated by the increased expression of $S O M$ and a decreased expression of Lig6. Moreover, MsZEP enhanced chlorophyll content in transgenic tobacco seedlings due to its function in xanthophyll cycle and ABA biosynthesis.

\section{References}

Agrawal, G.K., Yamazaki, M., Kobayashi, M., Hirochika, R., Miyao, A., Hirochika, H.: Screening of the rice viviparous mutants generated by endogenous retrotransposon tos 17 insertion. Tagging of a zeaxanthin epoxidase gene and a novel OsTATC gene. - Plant Physiol. 125: 1248-1257, 2001.

Audran, C., Liotenberg, S., Gonneau, M., North, H., Frey, A., Tap-Waksman, K., Vartanian, N., Marion-Poll, A.: Localisation and expression of zeaxanthin epoxidase mRNA in Arabidopsis in response to drought stress and during seed development. - Funct. Plant Biol. 28: 1161-1173, 2001.

Barrero, J.M., Piqueras, P., Gonzalez-Guzman, M., Serrano, R., Rodriguez, P.L., Ponce, M.R., Micol, J.L.: A mutational analysis of the ABA1 gene of Arabidopsis thaliana highlights the involvement of ABA in vegetative development. - J. exp. Bot. 56: 2071-2083, 2005.

Cutler, A.J., Krochko, J.E.: Formation and breakdown of ABA. Trends Plant Sci. 4: 472-478, 1999.

Frey, A., Audran, C., Marin, E., Sotta, B., Marion-Poll, A.: Engineering seed dormancy by the modification of zeaxanthin epoxidase gene expression. - Plant mol. Biol. 39: 1267-1274, 1999.

Frey, A., Boutin, J., Sotta, B., Mercier, R., Marion-Poll, A.: Regulation of carotenoid and ABA accumulation during the development and germination of Nicotiana plumbaginifolia seeds. - Planta 224: 622-632, 2006.

Grappin, P., Bouinot, D., Sotta, B., Miginiac, E., Jullien, M.: Control of seed dormancy in Nicotiana plumbaginifolia: post imbibition abscisic acid synthesis imposes dormancy maintenance. - Planta 210: 279-285, 2000.

Groot, S.P., Yperen, I.I., Van, Karssen, C.M.: Strongly reduced levels of endogenous abscisic acid in developing seeds of tomato mutant sitiens do not influence in vivo accumulation of dry matter and storage proteins. - Physiol. Plant. 81: 73-78, 1991.

Harmutk, L.: Chlorophyls and carotenoids: pigments of photosynthetic biomembranes. - Methods Enzimol. 148: 350382, 1987.

Holdsworth, M.J., Finch-Savage, W.E., Grappin, P., Job, D.: Post-genomics dissection of seed dormancy and germination. - Trends Plant Sci. 13: 7-13, 2008.

Je, J., Chen, H., Song, C., Lim, C.O.: Arabidopsis DREB2C modulates ABA biosynthesis during germination. - Biochem. biophys. Res. Commun. 452: 91-98, 2014.

Karssen, C.M., Brinkhorst-van der Swan, D.L., Breekland, A.E., Koornneef, M.: Induction of seed dormancy during seed development by endogenous abscisic acid: studies on abscisic acid-deficient genotypes of Arabidopsis thaliana L. Heynh. Planta 157:158-165, 1983.

Kim, D.H., Yamaguchi, S., Lim, S., Oh, E., Park, J., Hanada, A., Kamiya, Y., Choi, G.: SOMNUS, a CCCH-type zinc finger protein in Arabidopsis, negatively regulates light-dependent seed germination downstream of PIL5. - Plant Cell 20: 12601277, 2008.

Koornneef, M., Bentsink, L., Hilhorst, H.: Seed dormancy and germination. - Curr. Opin. Plant. Biol. 5: 33-36, 2002.
Lefebvre, V., North, H., Frey, A., Sotta, B., Seo, M., Okamoto, M., Nambara, E., Marion, P, A,: Functional analysis of Arabidopsis NCED6 and NCED9 genes indicates that ABA synthesized in the endosperm is involved in the induction of seed dormancy. - Plant J. 45: 309-319, 2006.

Linkies, A., Leubner-Metzger, G.: Beyond gibberellins and abscisic acid: how ethylene and jasmonates control seed germination. - Plant Cell Rep. 31: 253-270, 2012.

Marin, E., Nussaume, L., Quesada, A., Gonneau, M., Sotta, B., Hugueney, P., Frey, A., Marion-Poll, A.: Molecular identification of zeaxanthin epoxidase of Nicotiana plumbaginifolia, a gene involved in abscisic acid biosynthesis and corresponding to the ABA locus of Arabidopsis thaliana. - EMBO J. 15: 2331-2342, 1996.

Nambara, E., Marion-Poll, A.: Abscisic acid biosynthesis and catabolism. - Annu. Rev. Plant. Biol. 56: 165-185, 2005.

Park, H.Y., Seok, H.Y., Park, B.K., Kim, S.H., Goh, C.H., Lee, B.H., Lee, C.H., Moon, Y.H.: Overexpression of Arabidopsis ZEP enhances tolerance to osmotic stress. - Biochem. biophys. Res. Commun. 375: 80-85, 2008.

Rajjou, L., Duval, M., Gallardo, K., Catusse, J., Bally, J., Job, C., Job, D.: Seed germination and vigor. - Ann. Rev. Plant Biol. 63: 507-533, 2012.

Rock, C.D., Zeevaart, J.: The aba mutant of Arabidopsis thaliana is impaired in epoxy-carotenoid biosynthesis. - Proc. nat. Acad. Sci. USA. 88: 7496-7499, 1991.

Sasaki, K., Kim, M.-H., Kanno, Y., Seo, M., Kamiya, Y., Imai, R.: Arabidopsis COLD SHOCK DOMAIN PROTEIN 2 influences ABA accumulation in seed and negatively regulates germination. - Biochem. bioph. Res. Commun. 456: 380-384, 2015.

Thompson, A.J., Jackson, A.C., Parker, R.A., Morpeth, D.R., Burbidge, A., Taylor, I.B.: Abscisic acid biosynthesis in tomato: regulation of zeaxanthin epoxidase and 9-cisepoxycarotenoid dioxygenase mRNAs by light/dark cycles, water stress and abscisic acid. - Plant mol. Biol. 42: 833-845, 2000a.

Thompson, A.J., Jackson, A.C., Symonds, R.C., Mulholland, B.J., Dadswell, A.R., Blake, P.S., Burbidge, A., Taylor, I.B.: Ectopic expression of a tomato 9-cis-epoxycarotenoid dioxygenase gene causes over-production of abscisic acid. Plant J. 23: 363-374, 2000 b.

Tong, S.M., Xi, H.X., Ai, K.J., Hou, H.S.: Overexpression of wheat TaNCED gene in Arabidopsis enhances tolerance to drought stress and delays seed germination. - Biol. Plant. 61: 64-72, 2017.

Wang, N., Fang, W., Han, H., Sui, N., Li, B., Meng, Q.W.: Overexpression of zeaxanthin epoxidase gene enhances the sensitivity of tomato PSII photoinhibition to high light and chilling stress. - Physiol. Plant. 132: 384-396, 2008.

Waterworth, W.M., Masnavi, G., Bhardwaj, R.M., Jiang, Q., Bray, C.M., West, C.E.: A plant DNA ligase is an important determinant of seed longevity. - Plant J. 63: 848-860, 2010.

Wolters, A.M., Uitdewilligen, J.G., Kloosterman, B.A., Hutten, R.C., Visser, R.G., Van Eck, H.J.: Identification of alleles of carotenoid pathway genes important for zeaxanthin accumulation in potato tubers. - Plant mol. Biol. 73:659-671, 2010.

Xiong, L., Zhu, J.K.: Regulation of abscisic acid biosynthesis. Plant Physiol. 133: 29-36, 2003.

Yoshioka, T., Endo, T., Satoh, S., Restoration of seed germination at supraoptimal temperatures by fluridone, an inhibitor of abscisic acid biosynthesis. - Plant Cell Physiol. 39: 307-312, 1998.

Zhang, S.J., Song, G.Q., Li, Y.L., Gao, J., Liu, J.J., Fan, Q.Q., Huang, C.Y., Sui, X.X., Chu, X.S., Guo, D., Li, Y.: Cloning 


\section{Y.M. CAO et al.}

of 9-cis-epoxycarotenoid dioxygenase gene (TaNCED1) from wheat and its heterologous expression in tobacco. - Biol. Plant. 58: 89-98, 2014.

Zhang, Z., Wang, Y., Chang, L., Zhang, T., An, J., Liu, Y., Cao,
Y., Zhao, X., Sha, X., Hu, T.: MsZEP, a novel zeaxanthin epoxidase gene from alfalfa (Medicago sativa), confers drought and salt tolerance in transgenic tobacco. - Plant Cell Rep. 35: 439-453, 2016. 